\title{
A MAXIMALITY THEOREM IN FOURIER ANALYSIS
}

\section{ROBERT KAUFMAN}

Wermer's well-known maximality theorem [4], [5] has been generalized by Hoffman and Singer [2] as follows. Let $G$ be an ordered abelian group with nonnegative class $G_{+}$, and dual group $\Gamma$. Then the subalgebra $A$ of $C(\Gamma)$ generated by $G_{+}$can be extended to a maximal subalgebra of $C(\Gamma)$, provided there exists a homomorphism $\xi \not \equiv 0$ of $G$ in to the additive group $R$ with $\xi \leqq 0$ on $G_{+}$. The homomorphism $\xi$ is unique to within multiplication by a positive scalar, and the maximal subalgebras so obtained are translates of each other, as set forth precisely in [2]. (See [1, pp. 193-194] for an interesting investigation of maximality. $A$ is a Dirichlet algebra, [1].)

Our theorem is a converse: suppose that $B$ is a maximal proper closed subalgebra of $C(\Gamma)$, or of $l_{1}(G)$, and that for each element $g$ of $G$, either $g \in B$ or $g^{-1} \in B$. Of course, $l_{1}(G)$ is construed as a (dense) subalgebra of $C(\Gamma)$ via the Fourier transform.

TheOREM. There is a complex homomorphism $\Phi$ of $B$ with the property that $0<|\Phi| \leqq 1$ on $G \cap B$ and $|\Phi|$ is not identically 1 on $G \cap B$. In particular, if $A$ is contained in a maximal subalgebra of $C(\Gamma)$, the mapping $\log |\Phi|$ of $G_{+} \cap B$ can be extended to a negative mapping $\xi$ of $G$ into $R$.

Proof. Let $g_{1}, g_{2} \in G \cap B, g_{2}^{-1} \notin B$. We assert that for some $n \geqq 1$, $g_{2}^{n} g_{1}^{-1} \in B$. For otherwise, $g_{2}^{-n} g_{1} \in B$ for all $n \geqq 1$ and $g_{2}^{-n} g_{1}$ is singular, so the distance $\left\|1-g_{2}^{-n} g_{1} B\right\|=1$. Since the multiplications by $G$ are isometries of $l_{1}(G)$ and $C(\Gamma),\left\|g_{2}^{n}-g_{1} B\right\| \geqq 1$, for $n \geqq 1$. Applying the spectral radius formula to the algebra $B / g_{1} B$ we find a complex homomorphism $\Psi$ of $B$ for which $\Psi\left(g_{1}\right)=0,\left|\Psi\left(g_{2}\right)\right|=1$.

The homomorphism $\Psi$ admits an extension to the subalgebra generated by $B$ and $g_{2}^{-1}$, according to the rule $g_{2}^{m} x \rightarrow \Psi\left(g_{2}\right)^{m} \Psi(x)$, $x \in B,-\infty<m<\infty$. To see that this is bounded (and well-defined) note that

$$
\begin{aligned}
\left|\sum_{-N}^{N} \Psi\left(g_{2}\right)^{m} \Psi\left(x_{m}\right)\right| & =\left|\sum_{-N}^{N} \Psi\left(g_{2}\right)^{m+N} \Psi\left(x_{m}\right)\right| \\
& \leqq\left\|\sum_{-N}^{N} g_{2}^{m+N} x_{m}\right\|=\left\|\sum_{-N}^{N} g_{2}^{m} x_{m}\right\| .
\end{aligned}
$$

Received by the editors May 23, 1966. 
The algebra in question is larger than $B$ because it contains $g_{2}^{-1}$, and not dense because $g_{1}$ is singular in its closure. This contradiction proves our assertion.

Finally, let $g$ be any singular element of $G \cap B$. The spectrum of $g$ in $l_{1}(G)$, or $C(\Gamma)$, is just the unit circle, so that if $\lambda \in \mathrm{Sp}_{B} g$ and $|\lambda|<1$, then $\lambda$ is an interior point of $\mathrm{Sp}_{B} g$, by spectral permanence [3, pp. $33,142]$. Since $0 \in \mathrm{Sp}_{B} g$ this last set contains the entire unit disk. Then there is a homomorphism $\Phi$ of $B$ with $0<|\Phi(g)|<1$. Our assertion in the first paragraph shows that $0<\left|\Phi\left(g_{1}\right)\right| \leqq 1$ for all $g_{1} \in G \cap B$, because for some $n \geqq 1, g^{n} \in g_{1} B$. End of proof.

The weak direct sum . . $\oplus Z \oplus Z \oplus Z$ can be ordered lexicographically, and admits only the trivial positive mapping in to $R$. The algebra $A(\Gamma)$ is then included in no maximal subalgebra. The Gelfand space of $A(\Gamma)$ seems curious enough to reward a careful investigation.

\section{REFERENCES}

1. K. Hoffman, Banach spaces of analytic functions, Prentice-Hall, Englewood Cliffs, N. J., 1962.

2. K. Hoffman and I. M. Singer, Maximal subalgebras of $C(\Gamma)$, Amer. J. Math. 79 (1957), 295-305.

3. C. E. Rickart, Banach algebras, Van Nostrand, New York, 1960.

4. J. Wermer, Maximal subalgebras of group algebras, Proc. Amer. Math. Soc. 6 (1955), 692-694.

5. - On algebras of continuous functions, Proc. Amer. Math. Soc. 4 (1953), 866-869.

UNIVERSITY OF ILLINOIS 